\title{
Development, clinical utility, and place of ivacaftor in the treatment of cystic fibrosis
}

\author{
This article was published in the following Dove Press journal: \\ Drug Design, Development and Therapy \\ 29 August 2013 \\ Number of times this article has been viewed
}

\author{
Ruth O'Reilly \\ Heather E Elphick \\ Sheffield Children's Hospital, Western \\ Bank, Sheffield, United Kingdom
}

\begin{abstract}
Cystic fibrosis (CF) is a life-limiting, multisystem disease characterized by thick viscous secretions leading to recurrent lung infections, bronchiectasis, and progressive deterioration in lung function. $\mathrm{CF}$ is caused by loss or dysfunction of the CF transmembrane conductance regulator (CFTR) protein which is responsible for transepithelial chloride and water transport. Improved understanding of CFTR protein dysfunction has allowed the development of mutationspecific small-molecule compounds which directly target the underlying CFTR defect. Ivacaftor is the first licensed small-molecule compound for CF patients which targets the CFTR gating mutation Gly551Asp (previously termed G551D) and has the potential to be truly disease-modifying. Ivacaftor is an oral medication given twice daily and has shown benefit in terms of an increase in lung function, decreased sweat chloride, weight gain, improvement in patient-reported quality of life, and reduction in number of respiratory exacerbations in clinical trials. Although ivacaftor is currently only licensed for use in approximately $5 \%$ of the CF population (those who have at least one Gly551Asp mutation), the developmental pathway established by ivacaftor paves the way for other CFTR modulators that may benefit many more patients. In particular, a CFTR modulator for those with the Phe508del deletion (previously $\Delta F 508$ ) would allow $90 \%$ of the CF population to benefit from disease-modifying treatment.
\end{abstract}

Keywords: cystic fibrosis, cystic fibrosis transmembrane conductance regulator, ivacaftor

\section{Background}

Cystic fibrosis (CF) is the most common autosomal recessive disorder in Caucasians and affects approximately 28,000 patients in the United States and approximately 36,000 patients in Europe. ${ }^{1}$ It is a multisystem disease affecting primarily the lungs, causing thick viscous secretions and recurrent respiratory tract infections, but also affects the pancreas, hepatobiliary system, reproductive tract, gastrointestinal tract, and bones. Death is usually due to pulmonary damage from infections and inflammation (Figure 1).

Although the first clear description of CF was written in 1938 by Dr Dorothy Andersen, a pathologist in New York, the gene that encodes the CF transmembrane conductance regulator (CFTR) protein on the long (q) arm of chromosome 7 was only identified in $1989 .{ }^{2-4}$ More than 1900 CFTR gene mutations with the potential to cause disease have been described to date. ${ }^{5,6}$

The life expectancy of patients with CF has risen steadily over the last 25 years, from a median predicted age of survival of 25 years in 1985 to 37 years in 2011 . $^{7}$ Current treatment strategies in CF, including aggressive and early treatment of respiratory infections, mucociliary clearance, nutritional supplementation, and treatment
Correspondence: Heather Elphick Sheffield Children's NHS Foundation Trust, Western Bank, Sheffield, SIO 2TH United Kingdom

Tel +44 II 4 27| 7585

Fax +44 II4 27I 7672

Email heather.elphick@sch.nhs.uk 
CFTR gene mutation

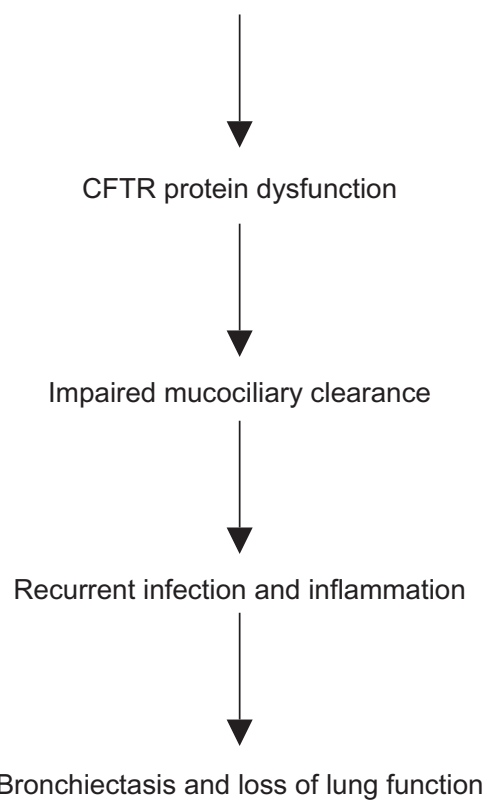

Figure I Model for cystic fibrosis lung disease.

Abbreviation: CFTR, CF transmembrane conductance regulator.

of airway inflammation, are associated with improved pulmonary function and reduced exacerbations. ${ }^{8}$ Until now, all treatments have targeted the downstream consequences of CFTR dysfunction rather than addressing the underlying CFTR defect. Ivacaftor, licensed in 2012, is the first available treatment to address directly the underlying genetic defect.

\section{Structure of CFTR protein}

The CFTR protein is a relatively large glycoprotein containing 1480 amino acids, ${ }^{4}$ with a molecular mass of $170,000 .{ }^{9}$ It is a member of the adenosine triphosphate (ATP) binding cassette family of proteins that are primarily concerned with transmembrane transport functions. ${ }^{4}$ The protein consists of two transmembrane regions, each linked to a nucleotide binding domain. ATP binds to both nucleotide binding domains to control gating of the channel. Spanning the region between nucleotide binding domain 1 and the second transmembrane region is a cytoplasmic regulatory domain (R-domain) which is comprised of many charged amino acids. The R-domain is a unique feature of CFTR. ${ }^{10}$ It contains several potential sites for phosphorylation by protein kinases. The activity of CFTR as an ion channel depends upon both phosphorylation of the R-domain and binding of ATP to the nucleotide binding domains.

Many of the mutations identified in $\mathrm{CF}$, including the most common mutation, Phe508del (previously $\Delta F 508$ ), occur in the first nucleotide binding domain 1 , while very few occur in the second. Around $75 \%$ of CF alleles contain the Phe $508 \mathrm{del}$ mutation, a three base pair deletion resulting in loss of a phenylalanine residue at position 508 in the protein.

\section{Function of CFTR protein}

CFTR functions as a regulated chloride ion channel and localizes to the apical cell membrane of secretory and absorptive epithelial cells within the airway, pancreas, liver, intestine, sweat gland, and vas deferens. ATP-driven conformational changes in CFTR open and close a gate to allow transmembrane flow of anions down their electrochemical gradient. ${ }^{11}$ There is also some evidence that CF lung disease relates to loss of regulation of the epithelial sodium channel transporter (ENaC) by CFTR, rather than being due just to chloride transport abnormalities. ${ }^{12,13}$

In $\mathrm{CF}$, dysfunctional CFTR protein leads to absent or decreased chloride secretion, increased sodium and water absorption, and liquid depletion on the surface of the airway. This causes thick viscous secretions and impairs mucociliary clearance, resulting in the classical manifestations of $\mathrm{CF}$, ie, infection, inflammation, and eventual bronchiectasis with loss of lung function. Sweat chloride levels are usually elevated $(>60 \mathrm{mmol} / \mathrm{L})$ in patients with $\mathrm{CF}$, whereas people without CF have sweat chloride levels $<40 \mathrm{mmol} / \mathrm{L}^{14}$

\section{Classification of CFTR mutations}

There are currently over 1900 known mutations affecting CFTR, many of which give rise to a disease phenotype. ${ }^{5}$ Six different classes are described. A brief description of the CFTR classification is described here and in Table 1.

Class I CFTR mutations affect biosynthesis of CFTR. They either prevent synthesis of a stable protein due to a nonsense mutation or result in production of a truncated protein

Table I CFTR protein defects and their potential therapies

\begin{tabular}{lll}
\hline Class & Consequence of mutation & $\begin{array}{l}\text { Mutation-specific } \\
\text { therapy }\end{array}$ \\
\hline II & No CFTR synthesis & $\begin{array}{l}\text { Ataluren (PTCI24) } \\
\text { VX-809 and ivacaftor } \\
\text { (combination) } \\
\text { to the cell surface }\end{array}$ \\
& & $\begin{array}{l}\text { VX-66I and ivacaftor } \\
\text { (combination) }\end{array}$ \\
III & Failure of CFTR to open & Ivacaftor \\
IV & at cell surface & \\
& Decreased time CFTR channel & \\
is open at cell surface & Decreased amount of CFTR & \\
VI & at cell surface & \\
& Unstable CFTR with decreased & \\
& half-life at the cell surface &
\end{tabular}

Abbreviation: CFTR, CF transmembrane conductance regulator. 
due to creation of a premature stop codon. These truncated proteins are unstable and are rapidly degraded by proteins in the endoplasmic reticulum. Class 1 mutations include the most severe phenotypes because no functional protein is synthesized. This type of mutation is found in $5 \%-10 \%$ of CF chromosomes worldwide, but account for around 60\% of mutations in Jewish people with $\mathrm{CF} .{ }^{15}$ The designation for nonsense mutation ends with an $\mathrm{X}$ and examples are Trp $1282 X$ (previously W1282X) and Gly542X.

Class II CFTR mutations affect CFTR maturation. They result in misprocessing of CFTR and give rise to misfolded CFTR protein, producing a lack of functional protein at the cell membrane. This altered protein fails to be trafficked to the correct location in the apical cell membrane, resulting in premature degradation by the proteasome. The small amount that does reach the correct location functions poorly. The Phe508del deletion accounts for the majority of mutations in this group. Around $90 \%$ of individuals with CF are heterozygous and $40 \%-50 \%$ are homozygous for the Phe $508 \mathrm{del} .{ }^{6,7}$

Class III CFTR mutations affect chloride channel regulation/gating. CFTR protein production and trafficking are normal, but when the protein arrives at the cell surface it does not respond to cAMP stimulation. This prevents binding of ATP and/or phosphorylation. The CFTR protein fails to open in response to intracellular signals at the cell surface, and remains closed for the majority of the time. The missense mutation Gly551Asp (previously termed G551D) is the third most common CFTR mutation and causes a glycineto-aspartate substitution at residue 551. ${ }^{7,16}$ Approximately $4 \%-5 \%$ of all patients with CF have the Gly551Asp mutation on at least one allele, ${ }^{6,7}$ although this mutation is more frequent in those of Celtic (Scottish, Irish, English) origin. ${ }^{17}$

Class IV CFTR mutations affect chloride conductance in the pore region of the channel. The CFTR protein reaches the cell surface, but the abnormal conformation of the pore leads to poor conductance of chloride ions because of reduced chloride permeation and open channel probability. Arg117His (previously termed $\mathrm{R} 117 \mathrm{H}$ ) is the most common class IV mutation, with a frequency of $2 \%-4 \%{ }^{6,7}$

Class V CFTR mutations affect mRNA stability. The splicing machinery is altered, resulting in generation of both aberrantly and correctly spliced mRNA. This leads to a reduced number of functioning CFTR proteins in the plasma membrane, as was demonstrated for the $3849+10 \mathrm{~kb} \mathrm{C}>\mathrm{T}$ mutation. ${ }^{18}$ Less than $1 \%$ of patients with $\mathrm{CF}$ have class $\mathrm{V}$ mutations. The level of functioning CFTR can vary between different patients and even between different organs of the same patient and often results in a milder clinical phenotype.
Class VI CFTR mutations affect CFTR stability. Truncation of the $\mathrm{C}$ terminus of CFTR produces a functional protein that is unstable at the apical membrane surface, eg, Gln1412X (previously termed Q1412X).

\section{Expression of the clinical phenotype}

The CF phenotype is highly heterogenous among individual patients, and even between siblings carrying identical CFTR mutations. Genotype-phenotype relationships are complex, and are affected by many factors, including alternative genomic loci containing modifier genes as well as environmental factors such as pollution, smoking, bacterial infection, malnutrition, and certain therapeutic agents. ${ }^{19}$ Of the various clinical symptoms of $\mathrm{CF}$, only pancreatic function has been shown to correlate well with CFTR genotype. ${ }^{20}$ In particular, substantial phenotypic variability is seen for lung disease, and there is often poor genotype-phenotype correlation for individual patients.

CFTR homozygous or compound heterozygous class I-III CFTR mutations are associated with an absence of or severely reduced production of functional CFTR. In general, this correlates well with a more severe phenotype involving pancreatic insufficiency ( $>95 \%$ of cases), liver disease $(3 \%-5 \%$ of cases), young age at diagnosis (usually less than one year), high sweat chloride levels ( $>80 \mathrm{mmol} / \mathrm{L})$, and meconium ileus (around $20 \%$ of cases). ${ }^{21-24}$ Class IV, V, and VI CFTR mutations are usually associated with some residual CFTR function and a more variable and sometimes milder disease with pancreatic sufficiency $(70 \%-80 \%$ of cases $)$, lower sweat chloride levels, no meconium ileus, and milder pulmonary disease. ${ }^{24}$ The class IV, V, and VI mutations usually dominate in patients who are compound heterozygotes.

The CFTR classification system has a number of limitations. Many of the mutations identified remain unclassified. ${ }^{5}$ In particular, the functional consequences of many rare mutations (particularly missense mutations) are unknown and cannot be predicted. Furthermore, a number of mutations give rise to more than one functional defect, eg, Phe508del, which may explain some of the clinical variability observed between subjects. ${ }^{25}$ The CFTR2 study is an international initiative that seeks to address this by providing expert-reviewed functional and clinical information on CFTR mutations. ${ }^{5,26}$

\section{Development of mutation- specific gene therapy}

After the CFTR gene was discovered in 1989, there was considerable hope that gene therapy could be rapidly developed. However, this has proven to be extremely challenging. 
There have been over 25 gene therapy trials using either viral or cationic lipid-based vector systems which have shown limited success. ${ }^{27}$ Even after successful uptake of the CFTR gene, the cells lose expression after a few weeks. Gene therapy, should it prove to be therapeutically effective, could be used to treat all patients with CF regardless of CFTR mutation. Currently, a gene therapy study is actively enrolling patients in the United Kingdom using a cationic lipid-based vector (GL67A/pGM169). ${ }^{28}$

An alternative to gene therapy, which shows more promise, is the development of small-molecule compounds that target specific CFTR mutations. These exciting new developments are based on improved understanding of CFTR structure and dysfunction in CF. Small-molecule compounds are divided into three groups, ie, CFTR correctors, CFTR potentiators, and CFTR suppressors. CFTR correctors facilitate the trafficking of more CFTR molecules to the airway epithelial surface. CFTR potentiators activate appropriately located mutant CFTR by increasing the time that activated CFTR channels at the cell surface remain open. CFTR suppressors of premature termination codons promote ribosomal read through of nonsense mutations. Each of these agents has therapeutic potential alone or in combination for a specific patient population. Small molecules that improve chloride ion transport in human bronchial CF cells are identified through high throughput screening which can simultaneously look at over 100,000 potential drugs. ${ }^{29,30}$ Molecules that look "active" on screening are then investigated further.

\section{Ivacaftor}

Ivacaftor (VX-770) is an oral, bioavailable potentiator that is designed to increase the time that activated CFTR channels at the cell surface remain open. It was licensed in 2012 both in the United States and Europe for use in patients with $\mathrm{CF}$ aged six years and over who carry at least one copy of the class III mutation, Gly551Asp. Ivacaftor is the first licensed CF medication actually addressing the primary consequences of CFTR dysfunction, rather than the downstream consequences of the disease, and is therefore potentially truly disease-modifying.

\section{Development}

Ivacaftor was identified by screening over 228,000 smallmolecule compounds using high throughput screening with a cell-based fluorescence membrane potential assay designed to identify CFTR potentiators. ${ }^{31}$ Initial in vitro studies of the effect of ivacaftor on CFTR-mediated chloride secretion have been performed on both recombinant cell lines and primary cultures of human bronchial epithelial cells. These studies have demonstrated that ivacaftor increases chloride transport by increasing the probability of the CFTR channel being open, and increases apical fluid height and ciliary beat frequency. ${ }^{31}$ The exact mechanism of action of ivacaftor at the CFTR channel is not completely understood. Further in vitro studies have elaborated on the mechanism further and have shown that ivacaftor opens the defective channel gate of mutant CFTR in a phosphorylation-dependent but ATP-independent manner. ${ }^{32}$

The efficacy and safety of ivacaftor in patients with $\mathrm{CF}$ with at least one Gly551Asp mutation in CFTR has been evaluated in two large, multicenter, randomized, double-blind, placebo-controlled trials. The subjects in these trials were clinically stable inpatients aged $>12$ years $(n=161)^{33}$ and children aged $6-12$ years $(n=52) .{ }^{34}$ The primary efficacy endpoint in both studies was the mean absolute change from baseline in forced expiratory volume in one second $\left(\mathrm{FEV}_{1}\right)$ percent predicted at 24 weeks from baseline. Patients in both trials were randomized in a 1:1 ratio to receive either $150 \mathrm{mg}$ of ivacaftor or placebo orally every 12 hours for 48 weeks, in addition to their usual CF therapies prescribed prestudy. ${ }^{33,34}$ In the adult and adolescent group, $70 \%$ of trial participants were on regular dornase alfa and $50 \%$ were on nebulized tobramycin. ${ }^{33}$ Use of inhaled hypertonic saline was not permitted because it does not have regulatory approval in the United States as a therapy for $\mathrm{CF}^{33}$ Ivacaftor was given with foods containing fat because this increases the exposure by $2-4$-fold. ${ }^{34}$ Patients from both studies were enrolled in a further open-label extension study for which interim results were available after a further 48 weeks for adults and adolescents and 24 weeks for children. ${ }^{35}$

The first of these studies recruited adults and adolescents (mean age 26 years) with $\mathrm{CF}$ and a mean $\mathrm{FEV}_{1}$ of $63.6 \%$ at entry to the study. The treatment effect of ivacaftor was an increase in $\mathrm{FEV}_{1}$ of $10.6 \%$. This was seen within two weeks of treatment and was sustained to week 48 and then to week 96 in the extended open-label study. ${ }^{33,34}$ Those treated with ivacaftor also had a 55\% decrease in respiratory exacerbations, a reduction in sweat chloride values (a measure of CFTR function) in the order of 50-60 mmol/L, and a weight gain of $2.7 \mathrm{~kg}$ more than in the placebo group. ${ }^{33}$ Improvements in quality of life as measured by the Cystic Fibrosis Questionnaire-Revised were also seen in those treated with ivacaftor. After the initial 24-week trial period, those who had been treated with placebo $(n=63)$ were started on treatment with ivacaftor. After 48 weeks on treatment, similar improvements in $\mathrm{FEV}_{1}$ of $9.4 \%$ were seen. ${ }^{34}$ 
The second study evaluated ivacaftor in 52 children (mean age nine years) who had a higher $\mathrm{FEV}_{1}$ (mean $84 \%$ ) than the previous group at entry to the study. ${ }^{34}$ Only four children had an $\mathrm{FEV}_{1}<70 \%$ at baseline. An overall ivacaftor treatment effect of $9.3 \%$ predicted $\mathrm{FEV}_{1}$ was shown. A smaller difference was shown between ivacaftor $(\mathrm{n}=11)$ and placebo $(\mathrm{n}=10)$ in the subgroup with a baseline $\mathrm{FEV}_{1}>90 \%(6.9 \%$ improvement). Improvements were sustained to week 72 in the open-label extension study. ${ }^{34}$ Although there were similar increases in weight and sweat chloride as in the adult study, no improvement was seen in the CF Quality of Life Questionnaire-Revised at either 24 or 48 weeks. ${ }^{34}$ Again, those children who were switched from placebo to ivacaftor $(n=22)$ in the follow-on open-label study showed a mean improvement in $\mathrm{FEV}_{1}$ of $8.1 \%$ after 24 weeks. ${ }^{34,35}$

The ivacaftor trials have highlighted the need for new clinical endpoints, particularly in children or older patients with mild lung disease. ${ }^{36}$ Although reductions in decline of $\mathrm{FEV}_{1}$ are often used as a measure of success in clinical trials, the sensitivity of this measure is limited in those with mild lung disease, such as the majority of the study group aged 6-12 years. ${ }^{37,38}$ This has provoked interest in the search for establishing reliable endpoints including sweat chloride, nasal potential difference, and intestinal current, all of which are surrogate markers of CFTR function. ${ }^{36}$

Ivacaftor is the first agent to show a reduction in the sweat chloride level to values below the diagnostic threshold for $\mathrm{CF}(60 \mathrm{mmol} / \mathrm{L}){ }^{33}$ Interestingly, in the trials, decreases in sweat chloride did not correlate with improvement in $\mathrm{FEV}_{1}$ in those treated with ivacaftor. ${ }^{39}$ Further, there was not a threshold level for change in sweat chloride above which an improvement in $\mathrm{FEV}_{1}$ was apparent. ${ }^{39}$ The lack of correlation of sweat chloride with improvement in $\mathrm{FEV}_{1}$ underlines the many physiological, environmental, and genetic factors that are likely to modulate CF disease severity and the difficulty in finding robust endpoints for clinical trials.

\section{Safety}

There are no identified adverse effects when ivacaftor is compared with placebo. However, although safety data are available for 60 weeks of treatment in the trials, the long-term safety profile of ivacaftor over a lifetime or its safety in young children is unknown. Most recently, an animal study showed that juvenile rats treated with ivacaftor from postnatal days $7-35$ at dose levels of $10 \mathrm{mg} / \mathrm{kg} /$ day and higher (approximately one tenth the maximum recommended human dose) developed cataracts. ${ }^{40}$ There is uncertainty about the relevance of this risk to children, given that there are differences in eye development between humans and rats. ${ }^{40} \mathrm{~A}$ further safety study is being conducted in children younger than 11 years of age (when eye development is usually complete) who will be followed up for at least two years with assessment at six-monthly intervals for both visual acuity and cataracts. The safety of ivacaftor in pregnancy and breast feeding has also not been established. As women remain healthier, with increased life expectancy and increased expectations from life, the safety of CFTR modulators in pregnancy needs to be considered.

\section{Clinical utility}

Current data suggest that ivacaftor could potentially prevent progressive lung damage and loss of lung function in patients with CF. Although it is unlikely that established structural lung damage will be reversed, correction of the chloride transport defect may allow aggressive symptomatic therapy to become more effective. Infants identified with the Gly551Asp mutation at newborn screening are most likely to benefit over their lifetime, potentially with a reduction in the need for other long-term treatments. The safety profile of ivacaftor in infants has not yet been established, but a trial of ivacaftor in children under six years of age with the Gly551Asp mutation is planned. However, starting ivacaftor therapy at birth will not prevent damage that is already present (such as congenital bilateral absence of the vas deferens and meconium ileus).

The financial viability of long-term use of ivacaftor is a concern, given that the cost per patient year in the United States is in the region of US $\$ 294,000 .{ }^{41}$ Although ivacaftor is currently licensed only for the Gly551Asp mutation, which affects less than $5 \%$ of the CF population, it represents a tremendous financial burden over a lifetime. It is also important to put in perspective that the increase in $\mathrm{FEV}_{1}$ and reduction in pulmonary exacerbations are similar to those seen in clinical trials for dornase alpha and azithromycin. ${ }^{42,43}$

\section{Other class III CFTR mutations}

In vitro data suggest that ivacaftor has a similar effect on other CFTR forms with gating defects (class III mutations) and support investigation of the potential clinical benefit of ivacaftor in CF patients who have CFTR gating mutations beyond Gly551Asp ${ }^{44}$ Extending the ivacaftor license further to other CFTR class III mutations (gating defects) would allow 10\%$15 \%$ of patients with CF to benefit from treatment. ${ }^{45}$ However, clinical studies in patients with individual class III mutations are difficult because of the low frequency at which many of these mutations occur. Expanding the pool of patients who are eligible for treatment with ivacaftor might be accomplished 
by allowing in vitro data or in vivo "n-of-1" studies (with, for example, reduction in sweat chloride concentration as an outcome measure) to validate the use of the drug. ${ }^{45}$

\section{Phe508del mutations}

A specific small-molecule compound or combination of compounds for Phe508del, the most common CFTR proteins mutation, would allow over $90 \%$ of the CF population to be treated with a disease-modifying therapy. ${ }^{6,7}$ Phe 508del CFTR proteins are largely destroyed in the endoplasmic reticulum, but channels that reach the cell surface do not open normally and are retrieved much faster than normal CFTR. In vitro studies have also shown that ivacaftor increases the activity of Phe508del CFTR channels, provided that they reach the cell surface. ${ }^{25}$

Ivacaftor monotherapy has been trialed in patients with Phe508del CFTR with much less success than in those with Gly551Asp. Efficacy results from an early clinical study in which patients homozygous for the Phe508del mutation $(\mathrm{n}=140)$ were randomized (4:1 ratio) to receive $150 \mathrm{mg}$ of ivacaftor every 12 hours or placebo for 16 weeks in addition to their prescribed CF therapies were disappointing. ${ }^{46}$ Treatment with ivacaftor showed no improvement in the primary outcome measure, ie, an increase in predicted $\mathrm{FEV}_{1}$ after 16 weeks, or in any secondary outcome measures, eg, weight gain or improvement in quality of life. ${ }^{46}$ There was no clinically meaningful change in sweat chloride levels, although there was a slight decrease $(2.9 \mathrm{mmol} / \mathrm{L})$ from baseline in the ivacaftor group. ${ }^{46}$

\section{In combination with other small- molecule compounds}

Another small-molecule compound, VX-809, a CFTR corrector, was developed after screening 164,000 small molecules for compounds that increased Phe508del CFTR-mediated chloride transport. ${ }^{47} \mathrm{VX}-809$ showed some efficacy in vitro by enhancing Phe508del CFTR processing and chloride transport in cultured human bronchial epithelial cells. ${ }^{47}$ However, the effect was much less than that seen with ivacaftor, and only $14 \%$ of epithelial cells showed improvement in chloride processing and secretion. ${ }^{47}$ Although the exact level of CFTR correction required to prevent or slow disease progression is unknown, it has been suggested that CFTR delivery to $25 \%$ of surface epithelial cells restores normal rates of mucus transport to $\mathrm{CF}$ airway epithelium. ${ }^{48}$ Early human studies with VX-809 monotherapy indicated a small dose-dependent effect on sweat chloride $(<10 \mathrm{mmol} / \mathrm{L})$ but no effect on lung function or patient-reported outcomes over the 28-day study period. ${ }^{49}$
However, it was hypothesized, based on in vitro data in cultured epithelial cells, that the combination of a CFTR corrector (VX-809) and a potentiator (ivacaftor) may be more effective than either agent alone. A VX-809-ivacaftor combination approach has progressed to Phase II investigation. Interim analysis of results from this ongoing Phase II trial in those homozygous for the Phe508del CFTR mutation are encouraging, demonstrating an $8.5 \%$ mean improvement in predicted $\mathrm{FEV}_{1}$ when compared with placebo. ${ }^{50}$ However, the decrease in sweat chloride was again small at $10 \mathrm{mmol} / \mathrm{L} .^{50}$ Secondly, there was a much smaller treatment effect on $\mathrm{FEV}_{1}$ predicted in those heterozygous for the Phe $508 \mathrm{del}$ mutation. ${ }^{50}$ Larger clinical trials are awaited.

An ongoing multicenter, double-blind, placebo-controlled Phase II trial is examining the therapeutic efficacy and safety of VX-661, another CFTR corrector, both alone and in combination with ivacaftor in adults homozygous for the Phe508del mutation. ${ }^{51}$ Initial reports have observed dose-dependent mean relative improvements of up to $9 \%$ in $\mathrm{FEV}_{1}$ predicted only in patients receiving the combination (VX-661 and ivacaftor) treatment at high doses versus placebo at day 28 , which returned to baseline during a 28-day post-washout treatment. ${ }^{52}$ There are plans to conduct further studies of VX-661 to evaluate further its potential for late-stage development, pending regulatory discussions.

\section{Small-molecule "CFTR suppressor" compounds}

Another small molecule compound, ataluren (PTC124), a CFTR suppressor, has been designed to overcome the adverse effects of a nonsense mutation by inducing selective ribosomal read through of mRNA containing a premature stop codon and allowing translation of a full length protein. ${ }^{53}$ Nonsense mutations are particularly common in Jewish communities, as previously stated. Ataluren is an orally bioavailable drug currently being investigated both in patients with $\mathrm{CF}$ and in those with Duchenne muscular dystrophy.

A small early clinical study in 23 patients from Israel with nonsense mutations involved taking ataluren three times daily for two weeks ( $4 \mathrm{mg} / \mathrm{kg}$ morning, $4 \mathrm{mg} / \mathrm{kg}$ midday, $8 \mathrm{mg} / \mathrm{kg}$ evening) followed by a washout period and a further two weeks at a higher dose $(10 \mathrm{mg} / \mathrm{kg}, 10 \mathrm{mg} / \mathrm{kg}, 20 \mathrm{mg} / \mathrm{kg}){ }^{54}$ Ataluren improved chloride ion transport as assessed by nasal potential difference (measuring the voltage across the nasal epithelium). ${ }^{54}$ In contrast, a previous trial with ataluren in 11 patients from the United States did not show electrophysiological improvement. ${ }^{55}$ Results from a larger, multinational, randomized, double-blind, placebo-controlled 
trial including both adults and children $(n=238)$ with $\mathrm{CF}$ who received ataluren $10 \mathrm{mg} / \mathrm{kg}$ (morning and midday) or $20 \mathrm{mg} / \mathrm{kg}$ (evening) or placebo has not shown a significant change in $\mathrm{FEV}_{1}$ or in respiratory exacerbations. ${ }^{56,57}$

Ataluren has also been trialed in boys with Duchenne muscular dystrophy, a genetic disease caused by a premature stop mutation in 5\%-13\% of cases. ${ }^{58}$ In early studies involving 38 boys with the disease who took ataluren for about a month, there were indications that muscle destruction had lessened, and dystrophin production increased in at least some trial participants. However, a larger randomized, multicenter, double-blind, placebo-controlled trial which followed 174 boys with Duchenne muscular dystrophy showed the primary endpoint of the six-minute walk test did not reach statistical significance. ${ }^{58}$

\section{Other potential treatments in clinical trials}

The knowledge base surrounding the structure and function of CFTR that has accumulated in the last 20 years continues to grow. Translational research feeding from this is now yielding compounds that provide real prospects of pharmacotherapy for this disease.

A major function of CFTR in healthy airways is to maintain an adequate liquid layer on the airway surface. In $\mathrm{CF}$, in addition to chloride channel dysfunction, defective regulation of epithelial sodium channels $(\mathrm{ENaC})$ leads to transport of salt and water out of the airways, exacerbating the reduction in airway surface liquid. Therefore, strategies designed to inhibit $\mathrm{ENaC}$ function may result in clinical benefit. A potential for treatment of $\mathrm{CF}$ is to use agents that inhibit $\mathrm{ENaC}$ either alone or as adjuncts to CFTR correctors and/or potentiators.

The possibility of using RNA interference to reduce expression of $\mathrm{ENaC}$ in the mouse lung has been investigated as a strategy to reduce sodium hyperabsorption in the $\mathrm{CF}$ lung. Potent ENaC alpha-targeted siRNA molecules capable of efficient knockdown of the $\mathrm{ENaC}$ alpha, beta, and gamma subunits have been demonstrated to inhibit the expression of ENaC alpha mRNA in murine and human cells grown in vitro and following in vivo delivery to the mouse lung. ${ }^{59}$ In addition, ENaC alpha-targeted shRNA can be used to knock down both human and mouse ENaC alpha mRNA. ${ }^{60}$ Further work is needed to assess the functional consequences of inhibiting $\mathrm{ENaC}$ in the lung.

Cell-based assays have shown improvements in trafficking and function of CFTR at the cell membrane with application of partial siRNA knockdown of the heat shock protein 90 cochaperone ATPase regulator Aha1. ${ }^{61}$ Histone deacetylase inhibitors and siHDAC7 may also be used to direct a global tissue response to Phe508del dysfunction that may ultimately improve surface hydration and reduce the inflammatory responses characteristic of the disease. ${ }^{62}$ A further way in which a pharmacological approach to $\mathrm{CF}$ can be considered is to recruit alternative chloride channels, such as calcium-activated chloride channel, to act as surrogates for CFTR. A number of P2Y2 receptor agonists have been investigated and found to operate by increasing $[\mathrm{Ca} 2+] \mathrm{i}$ which, in turn, activates calcium-activated chloride channels. Some of these compounds are currently undergoing clinical trials.

\section{Conclusion}

In the last two decades, significant progress has been made in the field of CFTR discovery, with improved understanding and the development of potentially disease-modifying treatments. Three new agents, ie, ivacaftor, VX-809, and ataluren, target the basic defects in production of CFTR. At present, ivacaftor has shown the greatest therapeutic promise and trials have shown beneficial effects on lung function, number of respiratory exacerbations, surrogate markers of CFTR function, nutrition, and quality of life. However, ivacaftor does have significant limitations in terms of cost and the current licensing agreement is only for those patients with a Gly551Asp mutation, which represents only around $5 \%$ of the CF population. Despite these limitations, ivacaftor is an exciting development which may pave the way for other CFTR-modulating treatments which may benefit more patients. At present, efforts to treat the Phe508del CFTR mutation with ivacaftor or other small-molecule compounds have not shown adequate therapeutic efficacy. Further trials are currently underway and their results are awaited. Importantly, as future therapies aim to prevent rather than improve existing organ damage, $\mathrm{FEV}_{1}$ is unlikely to be a sensitive enough endpoint for future trials. The reliability of other endpoints, even established measurements such as sweat chloride, need to be examined further as clinical endpoints.

\section{Disclosure}

The authors report no conflicts of interest in this work.

\section{References}

1. Farrell PM. The prevalence of cystic fibrosis in the European Union. J Cyst Fibros. 2008;7:450-453.

2. Kerem B, Rommens JM, Buchanan JA, et al. Identification of the cystic fibrosis gene: genetic analysis. Science. 1989;245:1073-1080.

3. Rommens JM, Iannuzzi MC, Kerem B, et al. Identification of the cystic fibrosis gene: chromosome walking and jumping. Science. 1989;245: 1059-1065. 
4. Riordan JR, Rommens JM, Kerem B, et al. Identification of the cystic fibrosis gene: cloning and characterization of complementary DNA. Science. 1989;245:1066-1073.

5. Cystic Fibrosis Mutation Database. CFTR1, 2013. Available from: http://www.genet.sickkids.on.ca/cftr/ StatisticsPage.html. Accessed May 16, 2013.

6. Cystic Fibrosis Registry Report Trust. Annual Data Report 2010, December 2011. Available from: http://www.cftrust.org.uk/aboutcf/ publications/cfregistryreports/UK_CF_Registry_-_Annual_Data_ Report_2010.pdf. Accessed May 16, 2013.

7. Patient Registry. Annual Data Report. Bethesda, MD: Cystic Fibrosis Foundation; 2011. Available from: http://www.cff.org/LivingWithCF/ QualityImprovement/PatientRegistryReport/. Accessed May 16, 2013.

8. Cohen-Cymberknoh M, Shoseyov D, Kerem E. Managing cystic fibrosis: strategies that increase life expectancy and improve quality of life. Am J Respir Crit Care Med. 2011;183:1463-1471.

9. Oxford University GeneMedicine. CFTR Protein Structure. Available from: http://www.genemedresearch.ox.ac.uk. Accessed April 20, 2013.

10. Bear CE, Li CH, Kartner N, et al. Purification and functional reconstitution of the cystic fibrosis transmembrane conductance regulator (CFTR). Cell. 1992;68:809-818.

11. Gadsby DC, Vergani P, Csanady L. The ABC protein turned chloride channel whose failure causes cystic fibrosis. Nature. 2006;440:477-483.

12. Knowles M, Gatzy J, Boucher R. Increased bioelectric potential difference across respiratory epithelia in cystic fibrosis. $N$ Engl J Med. 1981;305:1489-1495.

13. Mall M, Grubb BR, Harkema JR, O’Neal WK, Boucher RC. Increased airway epithelial $\mathrm{Na}+$ absorption produces cystic fibrosis-like lung disease in mice. Nat Med. 2004;10:487-493.

14. Farrell PM, Rosenstein BJ, White TB, et al. Guidelines for diagnosis of cystic fibrosis in newborns through older adults: Cystic Fibrosis Foundation consensus report. J Pediatr. 2008;153:S4-S14.

15. Kerem B, Chiba-Falek O, Kerem E. Cystic fibrosis in Jews: frequency and mutation distribution. Genet Test. 1997;1:35-39.

16. McKone EF, Emerson SS, Edwards KL, Aitken ML. Effect of genotype on phenotype and mortality in cystic fibrosis: a retrospective cohort study. Lancet. 2003;361:1671-1676.

17. Hamosh A, King TM, Rosenstein BJ, et al. Cystic fibrosis patients bearing both the common missense mutation Gly-Asp at codon 551 and the delta F508 mutation are clinically indistinguishable from delta F508 homozygotes, except for decreased risk of meconium ileus. Am J Hum Genet. 1992;51:245-250.

18. Highsmith WE, Burch LH, Zhou Z, et al. A novel mutation in the cystic fibrosis gene in patients with pulmonary disease but normal sweat chloride concentrations. N Engl J Med. 1994;331:974-980.

19. Kulczycki LL, Kostuch M, Bellanti JA. A clinical perspective of cystic fibrosis and new genetic findings: relationship of CFTR mutations to genotype-phenotype manifestations. Am J Med Genet A. 2003;116A: 262-267.

20. Davis PB, Schluchter MD, Konstan MW. Relation of sweat chloride concentration to severity of lung disease in cystic fibrosis. Pediatr Pulmonol. 2004;38:204-209.

21. McKone EF, Goss CH, Aitken ML. CFTR genotype as a predictor of prognosis in cystic fibrosis. Chest. 2006;130:1441-1447.

22. de Gracia J, Mata F, Alvarez A, et al. Genotype-phenotype correlation for pulmonary function in cystic fibrosis. Thorax. 2005;60:558-563.

23. Shoshani T, Augarten A, Gazit E, et al. Association of a nonsense mutation (W1282X), the most common mutation in the Ashkenazi Jewish cystic fibrosis patients in Israel, with presentation of severe disease. Am J Hum Genet. 1992;50:222-228.

24. Rowntree RK, Harris A. The phenotypic consequences of CFTR mutations. Ann Hum Genet. 2003;67:471-485.

25. Van Goor F, Straley KS, Cao D, et al. Rescue of DeltaF508-CFTR trafficking and gating in human cystic fibrosis airway primary cultures by small molecules. Am J Physiol Lung Cell Mol Physiol. 2006;290: L1117-L1130.
26. Clinical and Functional Translation of CFTR. CFTR2. Available from: http://www.cftr2.com. Accessed January 19, 2013.

27. Oakland M, Sinn PL, McCray PB Jr. Advances in cell and gene-based therapies for cystic fibrosis lung disease. Mol Ther. 2012;20:1108-1115.

28. The UK Cystic Fibrosis Gene Therapy Consortium. Gene therapy multi-dose clinical trial. Available from: http://www.cfgenetherapy. org.uk. Accessed April 20, 2013.

29. Carlile GW, Robert R, Zhang D, et al. Correctors of protein trafficking defects identified by a novel high-throughput screening assay. Chembiochem. 2007;8:1012-1020.

30. Galietta LV, Jayaraman S, Verkman AS. Cell-based assay for high-throughput quantitative screening of CFTR chloride transport agonists. Am J Physiol Cell Physiol. 2001;281:C1734-C1742.

31. Van Goor F, Hadida S, Grootenhuis PD, et al. Rescue of CF airway epithelial cell function in vitro by a CFTR potentiator, VX-770. Proc Natl Acad Sci U S A. 2009;106:18825-18830.

32. Eckford PD, Li C, Ramjeesingh M, Bear CE. Cystic fibrosis transmembrane conductance regulator (CFTR) potentiator VX-770 (ivacaftor) opens the defective channel gate of mutant CFTR in a phosphorylation-dependent but ATP-independent manner. J Biol Chem. 2012;287:36639-36649.

33. Ramsey BW, Davies J, McElvaney NG, et al. A CFTR potentiator in patients with cystic fibrosis and the G551D mutation. $N$ Engl J Med. 2011;365:1663-1672.

34. Vertex Pharmaceuticals. Kalydeco(C. Available from: http://www.ema. europa.eu/docs/en_GB/document_library. Acessed January 20, 2013.

35. [No authors listed]. Ivacaftor (Kalydeco) for cystic fibrosis. Med Lett Drugs Ther. 2012;54:29-30.

36. De Boeck K, Kent L, Davies J, et al. CFTR biomarkers: time for promotion to surrogate end-point. Eur Respir J. 2013;41:203-216.

37. Konstan MW, Wagener JS, Yegin A, Millar SJ, Pasta DJ, Vandevanter DR. Design and powering of cystic fibrosis clinical trials using rate of FEV(1) decline as an efficacy endpoint. J Cyst Fibros. 2010;9:332-338.

38. Que C, Cullinan P, Geddes D. Improving rate of decline of FEV1 in young adults with cystic fibrosis. Thorax. 2006;61:155-157.

39. Durmowicz AG, Witzmann KA, Rosebraugh CJ, Chowdhury BA. Change in sweat chloride as a clinical end point in cystic fibrosis clinical trials: the ivacaftor experience. Chest. 2013;143:14-18.

40. US Food and Drug Administration. Notification to the Cystic Fibrosis Community on Kalydeco (ivacaftor). Available from: http://www.fda. gov/Drugs/DrugSafety. Accessed April 20, 2013.

41. Bush A, Simmonds NJ. Hot off the breath: 'I've a cost for' - the 64 million dollar question. Thorax. 2012;67:382-384.

42. Fuchs HJ, Borowitz DS, Christiansen DH, et al. Effect of aerosolized recombinant human DNase on exacerbations of respiratory symptoms and on pulmonary function in patients with cystic fibrosis. The Pulmozyme Study Group. N Engl J Med. 1994;331:637-642.

43. Equi A, Balfour-Lynn IM, Bush A, Rosenthal M. Long term azithromycin in children with cystic fibrosis: a randomised, placebo-controlled crossover trial. Lancet. 2002;360:978-984.

44. Yu H, Burton B, Huang CJ, et al. Ivacaftor potentiation of multiple CFTR channels with gating mutations. J Cyst Fibros. 2012;11:237-245.

45. Davis PB, Yasothan U, Kirkpatrick P. Ivacaftor. Nat Rev Drug Discov. 2012;11:349-350.

46. Flume PA, Liou TG, Borowitz DS, et al. Ivacaftor in subjects with cystic fibrosis who are homozygous for the F508del-CFTR mutation. Chest. 2012;142:718-724.

47. Van Goor F, Hadida S, Grootenhuis PD, et al. Correction of the F508del-CFTR protein processing defect in vitro by the investigational drug VX-809. Proc Natl Acad Sci U S A. 2011;108:18843-18848.

48. Zhang L, Button B, Gabriel SE, et al. CFTR delivery to $25 \%$ of surface epithelial cells restores normal rates of mucus transport to human cystic fibrosis airway epithelium. PLoS Biol. 2009; 7:e1000155.

49. Clancy JP, Rowe SM, Accurso FJ, et al. Results of a phase IIa study of VX-809, an investigational CFTR corrector compound, in subjects with cystic fibrosis homozygous for the F508del-CFTR mutation. Thorax. 2012;67:12-18. 
50. Boyle M. The investigational CFTR corrector, VX-809, co-administered with the oral potentiator ivacaftor improved CFTR and lung function in F508del homozygous patients: Phase 2 study results. Poster 260 presented at the 26th North American Cystic Fibrosis Conference, Orlando, FL, October 11-13, 2012.

51. ClinicalTrials.gov. Study of VX-661 alone and in combination with VX-770 in subjects homozygous to the F508del-CFTR mutation. Available from: http://clinicaltrials.gov/show/NCT01531673. Accessed April 20, 2013.

52. Vertex. Treatment with VX-661 and ivacaftor in a Phase 2 study resulted in statistically significant improvements in lung function in people with cystic fibrosis who have two copies of the F508del mutation. Available from: http://investors.vrtx.com/releasedetail.cfm?releaseid=757597. Accessed April 20, 2013.

53. Welch EM, Barton ER, Zhuo J, et al. PTC124 targets genetic disorders caused by nonsense mutations. Nature. 2007;447:87-91.

54. Kerem E, Hirawat S, Armoni S, et al. Effectiveness of PTC124 treatment of cystic fibrosis caused by nonsense mutations: a prospective phase II trial. Lancet. 2008;372:719-727.

55. Clancy JP, Rowe SM, Bebok Z, et al. No detectable improvements in cystic fibrosis transmembrane conductance regulator by nasal aminoglycosides in patients with cystic fibrosis with stop mutations. Am J Respir Cell Mol Biol. 2007;37:57-66.
56. Kerem E, Wilschanski M, De Boeck K, et al. Phase 3 study of ataluren (PTC124 ${ }^{\circledR}$ ) in nonsense mutation cystic fibrosis (nmCF): baseline data. J Cyst Fibros. 2011;10:S17.

57. PTC Therapeutics Inc. Top-line data from phase 3 trial of ataluren in patients with nonsense mutation cystic fibrosis show promising results. Available from: http://ptct.Client.Shareholder.Com/releasedetail. Accessed January 26, 2013.

58. Mendell JR, Rodino-Klapac L, Sahenk Z, et al. Gene therapy for muscular dystrophy: lessons learned and path forward. Neurosci Lett. 2012;527:90-99.

59. Hyde SC, Painter H, Gill DR. EnaC knockdown in the mouse lung using RNAI. Pediatr Pulmonol. 2007;42:306.

60. Harding-Smith RE, Gill DR, Hyde SC. Knockdown of ENaCá, as a treatment for cystic fibrosis lung disease. Mol Ther. 2011;19:1360-1398.

61. Wang X, Venable J, LaPointe P, et al. Hsp90 cochaperone Aha1 downregulation rescues misfolding of CFTR in cystic fibrosis. Cell. 2006;127: 803-815.

62. Hutt DM, Herman D, Rodrigues AP, et al. Reduced histone deacetylase 7 activity restores function to misfolded CFTR in cystic fibrosis. Nat Chem Biol. 2010;6:25-33.
Drug Design, Development and Therapy

\section{Publish your work in this journal}

Drug Design, Development and Therapy is an international, peerreviewed open-access journal that spans the spectrum of drug design and development through to clinical applications. Clinical outcomes, patient safety, and programs for the development and effective, safe, and sustained use of medicines are a feature of the journal, which

\section{Dovepress}

has also been accepted for indexing on PubMed Central. The manuscript management system is completely online and includes a very quick and fair peer-review system, which is all easy to use. Visit http://www.dovepress.com/testimonials.php to read real quotes from published authors.

Submit your manuscript here: http://www.dovepress.com/drug-design-development-and-therapy-journal 